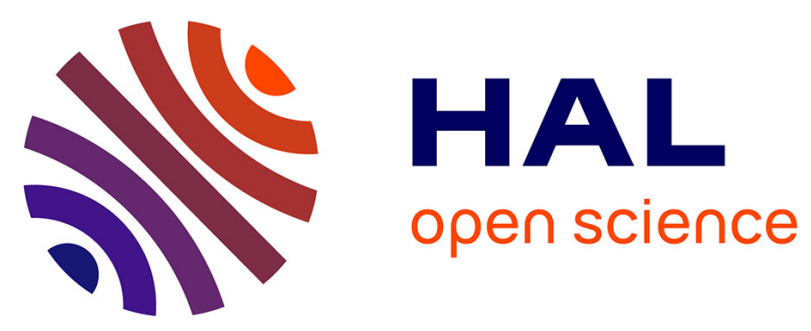

\title{
High Magnetic Field Generated by Bulk MgB2 Prepared by Spark Plasma Sintering
}

Kévin Berger, Michael Rudolf Koblischka, Bruno Douine, Jacques Noudem, Pierre Bernstein, Thomas Hauet, Jean Lévêque

\section{To cite this version:}

Kévin Berger, Michael Rudolf Koblischka, Bruno Douine, Jacques Noudem, Pierre Bernstein, et al.. High Magnetic Field Generated by Bulk MgB2 Prepared by Spark Plasma Sintering. IEEE Transactions on Applied Superconductivity, 2016, 26 (3), pp.6801005. 10.1109/TASC.2016.2537143 . hal01279399

\section{HAL Id: hal-01279399 \\ https://hal.science/hal-01279399}

Submitted on 26 Feb 2016

HAL is a multi-disciplinary open access archive for the deposit and dissemination of scientific research documents, whether they are published or not. The documents may come from teaching and research institutions in France or abroad, or from public or private research centers.
L'archive ouverte pluridisciplinaire HAL, est destinée au dépôt et à la diffusion de documents scientifiques de niveau recherche, publiés ou non, émanant des établissements d'enseignement et de recherche français ou étrangers, des laboratoires publics ou privés. 


\title{
High Magnetic Field Generated by Bulk $\mathrm{MgB}_{2}$ Prepared by Spark Plasma Sintering
}

\author{
Kévin Berger, Michael Rudolf Koblischka, Bruno Douine, \\ Jacques Noudem, Pierre Bernstein, Thomas Hauet and Jean Lévêque
}

\begin{abstract}
From the applications point of view, the advantage of low density given by $\mathrm{MgB}_{2}$ material must be taken into consideration and, the generation of strong magnetic flux densities using $\mathrm{MgB}_{2}$ should be investigated. In this contribution, we have studied the magnetic properties of samples processed by a fast Spark Plasma Sintering machine that is able to produce dense and high quality $\mathbf{M g B}_{2}$ samples. Experiments were carried out both on small pieces of the samples and on large-sized samples performing trapped field measurements in a FieldCooling process. For temperatures between $10 \mathrm{~K}$ to $30 \mathrm{~K}$, the results show a strong dependence of the magnetic behavior of the large-sized samples to the applied magnetic field sweep rate, while nothing particular appeared on the small samples. As the magnetic flux density produced by $\mathbf{M g B}_{2}$ bulks is directly linked to the potential of the applications, we report the field produced at the surface of one single $\mathrm{MgB}_{2}$ sample of $30 \mathrm{~mm}$ diameter and the field produced inside a stack of two $\mathrm{MgB}_{2}$ samples of $20 \mathrm{~mm}$ in diameter. A generation of magnetic flux density (magnetic polarization $\mu_{0} M$ ) up to $4.80 \mathrm{~T} @ 10 \mathrm{~K}$ and $3.92 \mathrm{~T} @ 20 \mathrm{~K}$ inside the stack of the two $\mathrm{MgB}_{2}$ samples was observed under negative supporting field of $-1.47 \mathrm{~T}$ and $-1.95 \mathrm{~T}$, respectively. According to these values, to their very low density and to their ease of manufacturing, $\mathrm{MgB}_{2}$ bulks are promising materials for the applications of superconductors.
\end{abstract}

Index Terms-Bulk $\mathrm{MgB}_{2}$, Field-cooling process, Flux jumps, Strong magnetic field, Trapped magnetic field.

\section{INTRODUCTION}

$I^{\mathrm{r}}$ $\mathrm{N}$ the field of High Temperature Superconductors (HTS), some applications requires the fabrication of large, bulk samples in order to employ them for levitation devices, fault current limiters, non-contact bearings for liquid pumping, magnetic shielding screens, and motors. $\mathrm{MgB}_{2}$ material

This work was supported in part by the French National Research Agency under Grand ANR-14-ASTR-0009 called RESUM for REalisation of a SUperconducting Motor.

Corresponding author: K. Berger (e-mail: kevin.berger@univ-lorraine.fr).

K. Berger, B. Douine and J. Lévêque are with the University of Lorraine, GREEN, Research Group in Electrical engineering and Electronics of Nancy EA 4366, Faculté des Sciences et Technologies, BP 70239, 54506 Vandoeuvre-lès-Nancy Cedex, France (e-mail: name.surname@univlorraine.fr)

T. Hauet is with the Institut Jean Lamour, Faculté des Sciences et Technologies, BP 70239, 54506 Vandoeuvre-lès-Nancy Cedex, France (thomas.hauet@univ-lorraine.fr)

M. R. Koblischka is with the Institute of Experimental Physics, Saarland University, Campus C63, 66123, Saarbrücken, Germany (e-mail: m.koblischka@mx.uni-saarland.de).

J. Noudem and P. Bernstein are with CRISMAT, CNRS/ENSICAEN, 6 Boulevard du Maréchal Juin, 14050 CAEN Cedex 4, France (jacques.noudem@ensicaen.fr, pierre.bernstein@ensicaen.fr). represents currently the metallic superconductor with the highest transition temperature [1]. A very promising feature of this material is that one can achieve a high critical current density in polycrystalline samples without aligning the crystal orientation with a substrate, which enables a much cheaper production technology [2]. Besides, different methods can be used for densifying the material such as Spark Plasma Sintering (SPS) process [3]. Using this process, $\mathrm{MgB}_{2}$ bulk samples with $99 \%$ of relative density have been obtained [4]. Theoretically, bulk $\mathrm{MgB}_{2}$ samples have very low density of $2.63 \mathrm{~g} / \mathrm{cm}^{3}$, compared to (RE) $\mathrm{BaCuO}$ samples which are around $6.36 \mathrm{~g} / \mathrm{cm}^{3}$, and $\mathrm{NdFeB}$ permanent magnets at around $7.60 \mathrm{~g} / \mathrm{cm}^{3}$. Practically, this ratio is maintained higher than $2-$ 3. From the applications point of view, this advantage must be taken into consideration and, the generation of strong magnetic flux densities using $\mathrm{MgB}_{2}$ should be investigated, even though $\mathrm{MgB}_{2}$ requires a lower operation temperature. Nowadays, with developed cryocooling systems, this is not a large obstacle anymore.

In this contribution, we studied the magnetic properties of samples with a density of $2.61 \mathrm{~g} / \mathrm{cm}^{3}$, processed at CRISMAT by a fast SPS machine that is able to produce dense and high quality $\mathrm{MgB}_{2}$ samples. The process, the structural properties and the superconducting transition characteristics of the obtained bulks were reported previously [4]. Experiments were carried out both on small pieces of the samples and on large-sized samples performing trapped field measurements in a Field Cooling (FC) process.

\section{SAMPLES CHARACTERIZATION}

Magnetic characterization of a small sample piece of $1.377 \mathrm{~mm} \times 1.473 \mathrm{~mm} \times 0.638 \mathrm{~mm}$ was carried out at $\mathrm{IJL}$ Nancy using a Magnetic Property Measurement System (MPMS $® 3$ ) from Quantum Design.

The DC magnetic moment $m$ was measured at $10 \mathrm{~K}, 20 \mathrm{~K}$ and $30 \mathrm{~K}$, for an applied field parallel to the longest dimension of the sample. Half of the Major Hysteresis Loops (MHL) of the magnetic moment $m$ are shown in Fig. 1. The field was cycled between $+5 \mathrm{~T}$ and $-5 \mathrm{~T}$ with various sweep rates: $1 \mathrm{mT} / \mathrm{s}, 4 \mathrm{mT} / \mathrm{s}$ and $20 \mathrm{mT} / \mathrm{s}$. In this range, with these small samples, no phenomena of flux jumps and no influence of the sweep rate were observed.

\section{A. Determination of $J_{\mathrm{c}}(B)$}

The critical current density $J_{\mathrm{c}}$ is calculated from the Major Hysteresis Loop (MHL) for which the sample is in the critical 


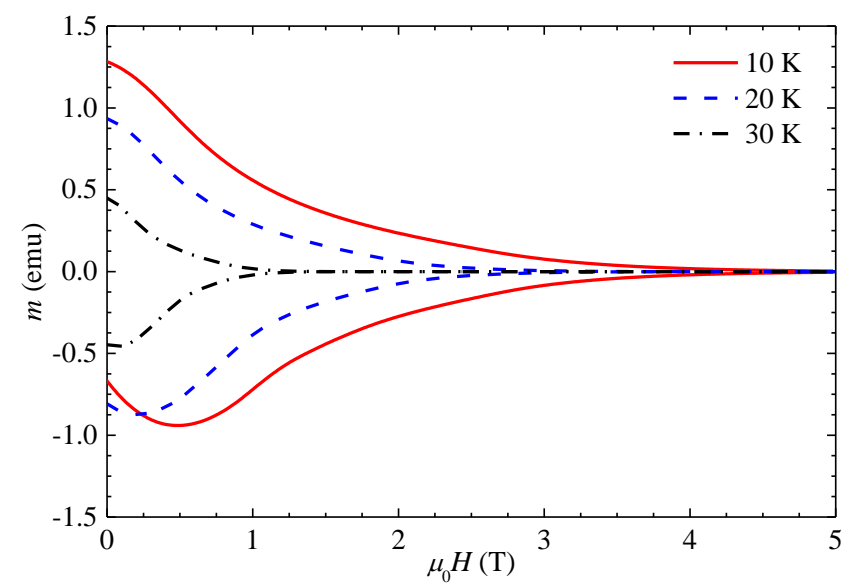

Fig. 1. Half of the Major Hysteresis Loops of the magnetic moment $m$ of a small $\mathrm{MgB}_{2}$ sample measured at $10 \mathrm{~K}, 20 \mathrm{~K}$ and $30 \mathrm{~K}$, for an applied field cycled between $+5 \mathrm{~T}$ and $-5 \mathrm{~T}$.

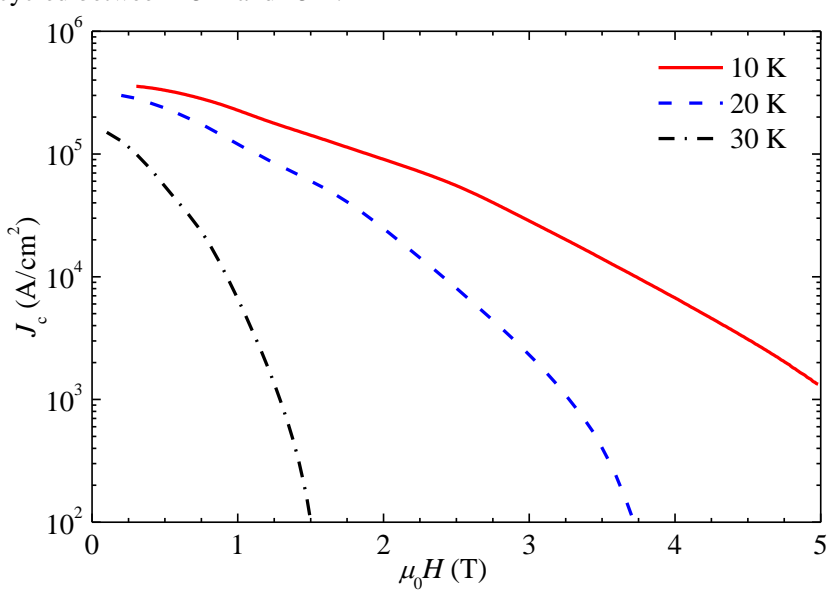

Fig. 2. Field dependence of the critical current density calculated at $10 \mathrm{~K}$, $20 \mathrm{~K}$ and $30 \mathrm{~K}$ from the MHL in Fig. 1.

state. In the case of an infinitely long sample with a rectangular cross-section $(2 a \times 2 b$, with $a<b)$, the critical current is given by

$$
J_{\mathrm{c}}(B)=\Delta M(a(1-a / 3 b))^{-1}
$$

where $\Delta M$ is the width of the curve [5], [6].

It can be noticed that due to some uncertainty in the size measurements of the sample and its volume estimation, the value of the critical current density may be overestimated by $5 \%$. Nevertheless, the critical current densities at $10 \mathrm{~K}$ and $20 \mathrm{~K}$ exhibit values higher than $10^{5} \mathrm{~A} / \mathrm{cm}^{2}$ for $1 \mathrm{~T}$ of applied field, what is a relatively good result without doping [7].

\section{B. Irreversibility field $H_{\text {irr }}$}

The irreversibility field was determined at the point where $J_{\mathrm{c}}$ meets the criterion of $100 \mathrm{~A} / \mathrm{cm}^{2}$. This irreversibility field is greater than $5 \mathrm{~T}$ at $10 \mathrm{~K}$, and equal to $3.71 \mathrm{~T}$ and $1.50 \mathrm{~T}$, respectively at $20 \mathrm{~K}$ and $30 \mathrm{~K}$.

\section{EXPERIMENTAL SETUP}

\section{A. Apparatus}

The cryostat employed for the trapped field measurements in our case is an Oxford Instruments $5 \mathrm{~T}$ low-loss cryostat with a room-temperature bore. On removing the secondary cryostat for sample cooling used for low-temperature STM

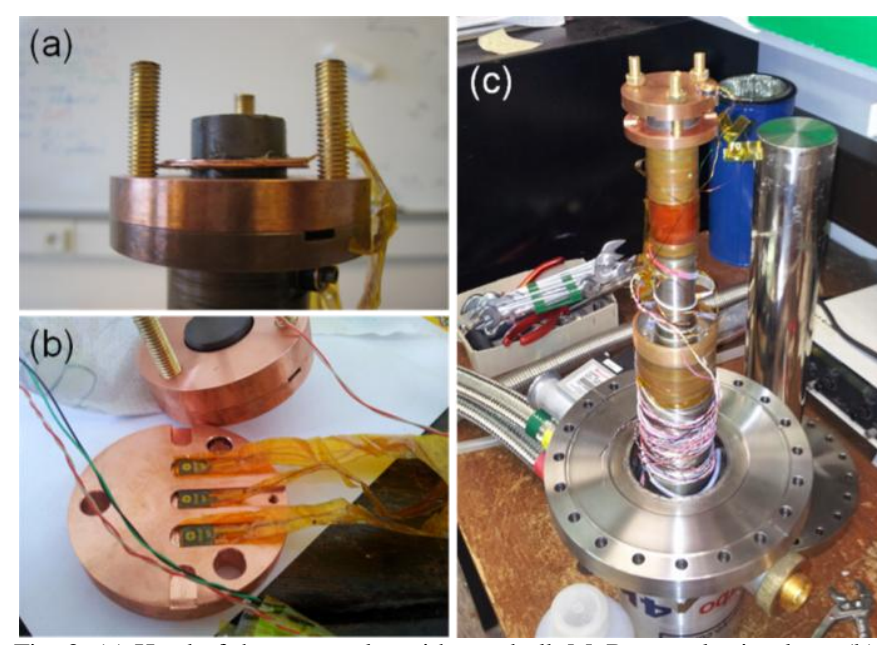

Fig. 3. (a) Head of the cryocooler with two bulk $\mathrm{MgB}_{2}$ samples in place. (b) Copper top-plate with slits for three Hall probes spaced by $1 \mathrm{~cm}$. (c) The entire cryocooler system with a mounted sample.

and MFM work, the room-temperature bore of $7.5 \mathrm{~cm}$ diameter is fully exposed. Fitting a cryocooler under it enables trapped-field experiments to be performed in magnetic fields up to $\pm 5 \mathrm{~T}$ perpendicular to the sample surface. For our experiments, we have employed the ARS-4K-cryocooler system, which can reach temperatures down to $4.2 \mathrm{~K}$ but the lowest temperature in our experiments is roughly $9 \mathrm{~K}$.

A bi-directional power supply for the superconducting coil (Oxford Instruments IPS 120-10) enables a continuous field sweep through $0 \mathrm{~T}$ together with a controlled magnetic fieldsweep rate ranging up to $40 \mathrm{mT} / \mathrm{s}(2.4 \mathrm{~T} / \mathrm{min})$.

Up to 3 Hall probes may be employed to measure the fields at the sample surface. We have used high linearity Hall probes HHP-NP from Arepoc which have a sensibility higher than $70 \mathrm{mV} / \mathrm{T}$. The overall dimensions of the Hall probe are $7 \mathrm{~mm} \times 5 \mathrm{~mm} \times 1 \mathrm{~mm}$ and the active area is approximately $0.35 \mathrm{~mm}$ far from the sensor surface. The calibrations of these sensors were performed at each mounting of a sample.

Fig. 3 (a) presents the sample stage of the cryocooler with two bulk $\mathrm{MgB}_{2}$ samples in place. There are copper discs between the 2 samples in order to connect them thermally despite the presence of the Hall probes. In Fig. 3 (b), a copper ring is fitted around the sample for better temperature exchange during the cooling, and another copper plate is then fixed above the sample containing three slits for the Hall probes. Finally, Fig. 3(c) shows the entire cryocooler head with a sample and the Hall probes in place. In this way, samples up to $4 \mathrm{~cm}$ in diameter and $3 \mathrm{~cm}$ in length can be cooled and submitted to a high magnetic field.

\section{B. Large-sized $\mathrm{MgB}_{2}$ samples}

Three large-sized $\mathrm{MgB}_{2}$ samples have been used in our trapped field measurements. The height $h$ of each of them is $9.7 \mathrm{~mm}$ and they have been made using a SPS process with a heat treatment at $1200{ }^{\circ} \mathrm{C}$ under $50 \mathrm{MPa}$ pressure.

One sample, called hereafter $d 30$, with a diameter $d$ of $30 \mathrm{~mm}$, has been reacted during $15 \mathrm{~min}$. The two others samples, named $d 20$, with a diameter of $20 \mathrm{~mm}$ have been reacted during $10 \mathrm{~min}$ each. 


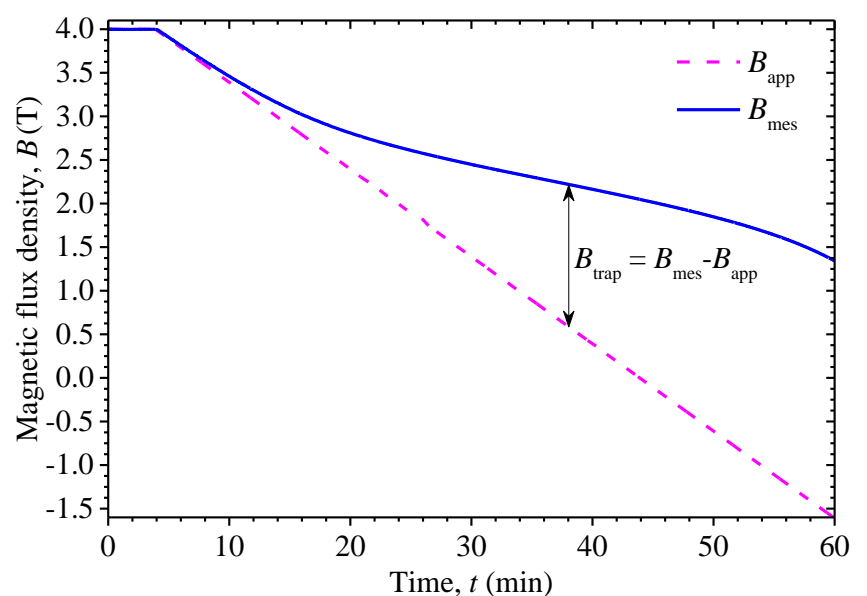

Fig. 4. Illustration of a Field Cooling (FC) process at $4 \mathrm{~T}$ and $20 \mathrm{~K}$ on the sample $d 30$. The difference between the measured field $B_{\text {meas }}$ and the applied field $B_{\text {app }}$ is defined as the trapped magnetic field $B_{\text {trap }}$.

\section{TRAPPED FIELD MEASUREMENTS}

In the following, we present the trapped field measurements on the large-sized bulk $\mathrm{MgB}_{2}$ samples.

\section{A. Field-cooling process}

To activate/energize the $\mathrm{MgB}_{2}$ pellets, we employ the FieldCooling (FC) process. For this purpose, we tried also the use of different field sweep rates in order to see the dependence on potential flux jumps of this sweep rate. An example of FC process on the sample $d 30$ is illustrated in Fig. 4. Here, a magnetic field of $4 \mathrm{~T}$ is applied to the sample which is then cooled down below its critical temperature. The applied magnetic field is then decreased with a fixed sweep rate. The difference between the measured field $B_{\text {meas }}$ and the applied field $B_{\text {app }}$ is defined as the trapped magnetic field $B_{\text {trap }}$, this is the reaction of the sample. This definition of $B_{\text {trap }}$ also corresponds to the magnetic polarization of the HTS sample $\mu_{0} M$. The remanent trapped magnetic field $B_{\text {rem }}$ is defined when the applied field is zero. The definition of the trapped magnetic field $B_{\text {trap }}$ is essential, since only the remanent trapped magnetic field is usually reported. However for practical applications as in [8]-[10], higher values of $B_{\text {trap }}$ are of great importance. Indeed, for some kinds of high power density superconducting motors, the output power is directly dependent on the magnetic flux density that can produce HTS bulks. For example, if some HTS bulks, cooled under a field of $3 \mathrm{~T}$ and then submitted to a negative field of $-3 \mathrm{~T}$, can maintain the initial value of $3 \mathrm{~T}$, this means that they can generate $6 \mathrm{~T}$ thanks to the current density induced inside.. This is relevant value for such applications.

\section{B. One single $\mathrm{MgB}_{2}$ sample}

In this section, we report the performances in terms of trapped magnetic field of one single $\mathrm{MgB}_{2}$ sample.

Fig. 5 shows the magnetic field trapped at the center of the surface of the sample $d 30$ at $10 \mathrm{~K}$ and $20 \mathrm{~K}$. At $20 \mathrm{~K}$, there are less flux jumps when the field sweep rate is $0.10 \mathrm{~T} / \mathrm{min}$ instead of $0.50 \mathrm{~T} / \mathrm{min}$. This sweep rate influence on the flux jumps has also been observed in [11]. The remanent magnetic

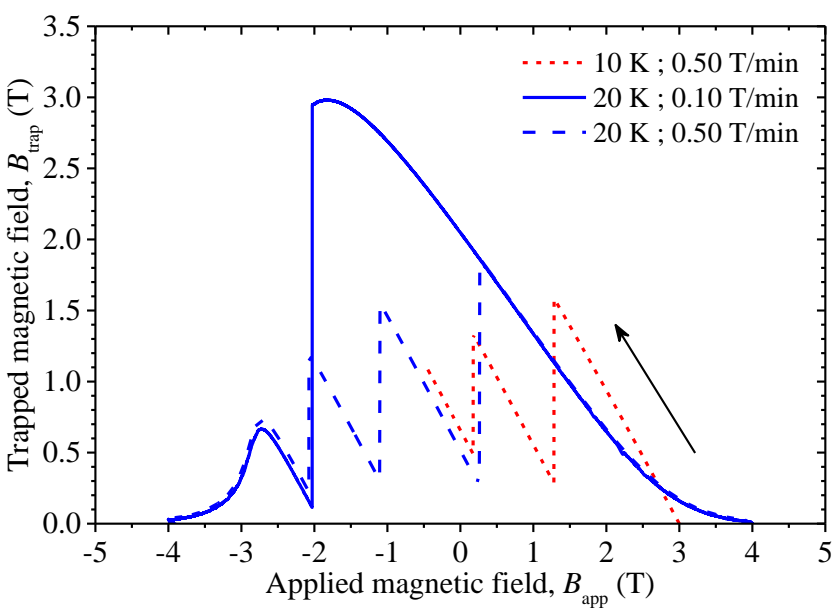

Fig. 5. Magnetic field trapped at the center of the surface of the sample $d 30$ at $10 \mathrm{~K}$ and $20 \mathrm{~K}$ after field cooling at $3 \mathrm{~T}$ or $4 \mathrm{~T}$. At $20 \mathrm{~K}$, there are less flux jumps when the field sweep rate is $0.10 \mathrm{~T} / \mathrm{min}$ instead of $0.50 \mathrm{~T} / \mathrm{min}$.

field is equal to $2.05 \mathrm{~T}$ at $20 \mathrm{~K}$ and the highest value of trapped/produced magnetic field by this sample is around $2.98 \mathrm{~T}$ at $20 \mathrm{~K}$. Similar values around $2 \mathrm{~T}$ have been recently summarized for the same sample size and at the same temperature in Table 1 of [12].

Given that many flux jumps occurred at $10 \mathrm{~K}$, even at low sweep rate, it was not possible to perform high trapped field at this temperature. More details concerning the flux jumps in $\mathrm{MgB}_{2}$ can be found in [13].

\section{Two $\mathrm{MgB}_{2}$ samples stacked together}

In this section, we report the performances in terms of trapped magnetic field of two $\mathrm{MgB}_{2}$ samples stacked together. Because we did not have two samples of $30 \mathrm{~mm}$ of diameter, we used samples of same properties but with a diameter of $20 \mathrm{~mm}$, that is the two $d 20$ samples. The two $d 20$ pellets are separated by an air gap of the size of the Hall probe, i.e. $1 \mathrm{~mm}$. By neglecting this air gap, it can be considered that the stack forms a single $\mathrm{MgB}_{2}$ pellet of $20 \mathrm{~mm}$ of diameter and roughly $20 \mathrm{~mm}$ height, that will be later called $d 20 \times 2$.

Fig. 6 shows the trapped magnetic field results on a stack of two $\mathrm{MgB}_{2}$ samples $d 20 x 2$ at $10 \mathrm{~K}, 20 \mathrm{~K}$ and $25 \mathrm{~K}$ (a) at the center of the surface of the stack, and (b) at the center between the two samples. As expected, the values shown in (b) are higher than the one in (a), but the shape of the curves is the same. Some flux jumps appears at $20 \mathrm{~K}$ with $0.50 \mathrm{~T} / \mathrm{min}$ and $10 \mathrm{~K}$ with $0.05 \mathrm{~T} / \mathrm{min}$. Unfortunately, it was not possible to repeat measurements at $10 \mathrm{~K}$ with lower sweep rates in order to remove the flux jump.

Fig. 7 shows a bar graph synthetising the results of maximum trapped magnetic field using $d 20 \times 2$. The red bars (left) correspond to the measures at the center of the top surface of the $d 20 \times 2$ assembly, whereas the black ones (right) correspond to the measures inside $d 20 \times 2$, in the air gap. The corresponding values are directly written above the bars. The hashed part of the bars represents the remanent trapped magnetic field $B_{\text {rem, }}$, when the applied field is zero, whereas the full bars are associated to the maximum trapped field $B_{\text {trap }}$ achieved in our experiments. Similar results on others samples have been reported in [14], [15]. From Fig. 1 of [14], we can 


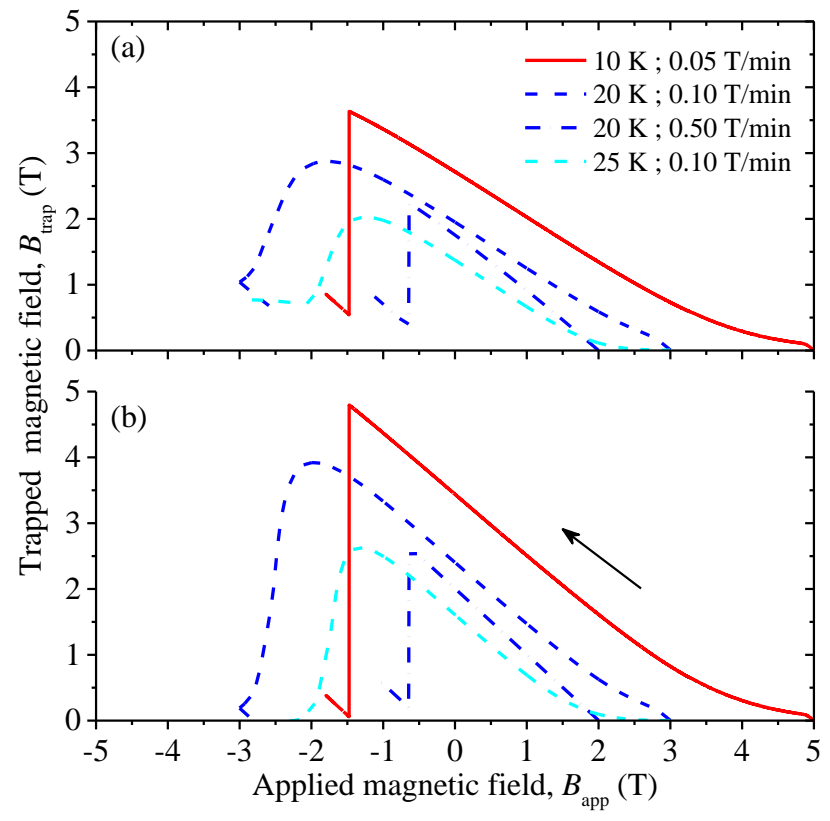

Fig. 6. Trapped magnetic field results on a stack of two $\mathrm{MgB}_{2}$ samples $d 20 \times 2$ at $10 \mathrm{~K}, 20 \mathrm{~K}$ and $25 \mathrm{~K}$ (a) at the center of the surface of the stack, (b) at the center between the two samples.

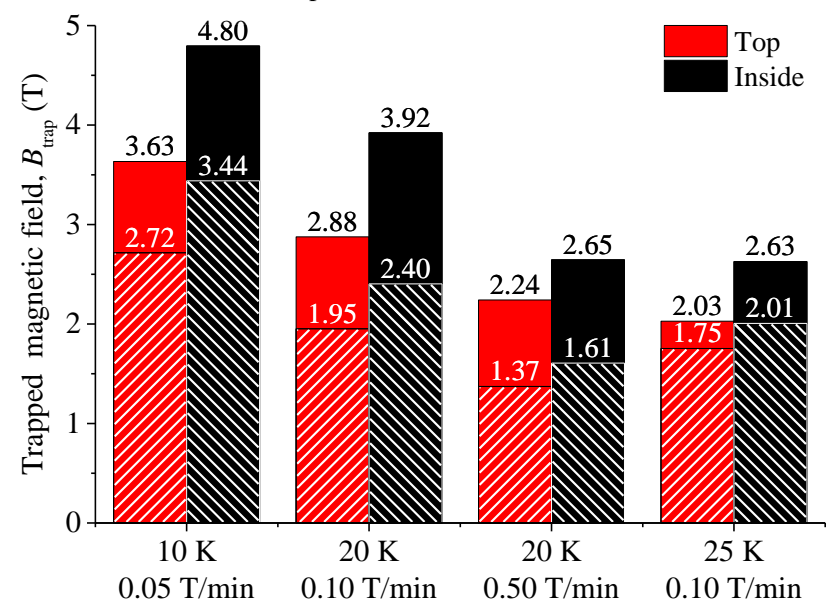

Fig. 7. Bar graph synthetising the results of maximum trapped magnetic field using $d 20 \times 2$. The hashed part of the bars represents the remanent trapped magnetic field $B_{\text {rem, }}$, whereas the full bars are associated to the maximum trapped field $B_{\text {trap }}$.

deduce the maximal trapped magnetic field $B_{\text {trap }}$ inside the bulk disk pair: $6.25 \mathrm{~T}, 4.66 \mathrm{~T}$, and $2.21 \mathrm{~T}$, at $10 \mathrm{~K}, 20 \mathrm{~K}$ and $30 \mathrm{~K}$, and under negative supporting field of $-3 \mathrm{~T},-2.23 \mathrm{~T}$ and $-1 \mathrm{~T}$, respectively. A value of $B_{\text {rem }}$ equal to $2.60 \mathrm{~T}$ at $20 \mathrm{~K}$ has also been measured inside a disk pair in [15].

\section{RESULTS AND DISCUSSION}

It is obvious that the remanent magnetic field anywhere in the sample cannot be higher than the irreversibility field reported in Section II.B. The irreversibility field values of our samples prepared by SPS are clearly lower than those reported in [12] for samples prepared by hot-pressing of ball-milled precursor powder, where the irreversibily fields are about $7 \mathrm{~T}$ at $20 \mathrm{~K}$ and $2 \mathrm{~T}$ at $30 \mathrm{~K}$.

According to [16]-[18], the external magnetic field necessary for full flux penetration at the center of the sample
TABLE I

COMPARISON OF THEORETICAL AND EXPERIMENTAL RATIO $B_{\mathrm{P}} / B_{\mathrm{P}}$

\begin{tabular}{lcccc}
\hline \hline Ratio $B_{\mathrm{p}} / B_{\mathrm{p}}{ }^{\mathrm{s}}$ & $10 \mathrm{~K}$ & $20 \mathrm{~K}$ & $20 \mathrm{~K}$ & $25 \mathrm{~K}$ \\
& $0.05 \mathrm{~T} / \mathrm{min}$ & $0.10 \mathrm{~T} / \mathrm{min}$ & $0.50 \mathrm{~T} / \mathrm{min}$ & $0.10 \mathrm{~T} / \mathrm{min}$ \\
\hline Theoretical & 1.82 & 1.82 & 1.82 & 1.82 \\
With $B_{\text {trap }}^{\max }$ & 1.32 & 1.36 & 1.18 & 1.30 \\
With $B_{\text {rem }}$ & 1.26 & 1.23 & 1.18 & 1.15 \\
\hline \hline
\end{tabular}

can be written as

$$
B_{\mathrm{p}}=\mu_{0} J_{\mathrm{c}}^{\text {bulk }} R f\left(k_{d}\right) \text {, with } f\left(k_{d}\right)=k_{d} \ln \left(\frac{1+\sqrt{1+k_{d}^{2}}}{k_{d}}\right)
$$

with $J_{\mathrm{c}}^{\text {bulk }}$ as the field-independent bulk critical current density, $R$ the radius, $d$ the diameter and $h$ the height of the sample, and $k_{d}=h / d$. Since $B_{\mathrm{p}}$ corresponds to the full penetration field, it gives an idea of the achievable trapped magnetic flux density at the center of the sample. However, (2) is based on the hypothesis of a constant critical current density and does not take into account the $J_{c}(B)$ law of the material. Using the same hypotheses, one can also express the corresponding field at the center of the surface of the sample, see (12) in [18]:

$$
B_{\mathrm{p}}^{\mathrm{s}}=\frac{\mu_{0} J_{\mathrm{c}}^{\text {bulk }} R}{2} f\left(k_{R}\right), \text { with } k_{R}=2 k_{d}=h / R
$$

In that case, the ratio $B_{\mathrm{p}} / B_{\mathrm{p}}{ }^{\mathrm{s}}$ is only driven by geometric parameters. For the samples studied here, this ratio is equal to $1.51,1.63$ and 1.82 for $d 30, d 20$ and $d 20 \times 2$, respectively. As $\lim _{k \rightarrow \infty} f(k)=1$, a 'half' infinite long cylinder would correspond to a ratio $B_{\mathrm{p}} / B_{\mathrm{p}}{ }^{\mathrm{s}}$ of 2 . However, extrapolating the value of the trapped magnetic field at the center of the sample, as some authors do, by multiplying the value of the trapped magnetic field at the surface with the theoretical ratio $B_{\mathrm{p}} / B_{\mathrm{p}}{ }^{\mathrm{s}}$ is not sufficient, and leads to overestimated values. The comparison is made in Table I for $d 20 x 2$. Given the fact that the only relevant value for the practical applications as permanent magnet is the trapped magnetic field at the surface of the sample, the value of the trapped field inside the sample is usually reported for comparison reasons or in order to impress.

From (2) and (3), one can say that the trapped magnetic field, for a fixed height of the sample, can be increased simply by increasing the diameter of the sample. Theoretically, choosing $d 30$ instead of $d 20$ leads to $26 \%$ and $35 \%$ of increase for $B_{\mathrm{p}}$ and $B_{\mathrm{p}}{ }^{\mathrm{s}}$, respectively. In the same way, choosing $d 20 \times 2$ instead of $d 20$ leads to $23 \%$ and $10 \%$ of increase for $B_{\mathrm{p}}$ and $B_{\mathrm{p}}^{\mathrm{s}}$, respectively. Therefore, the increase of the trapped magnetic field at the surface of the sample is more significant by increasing the diameter from $20 \mathrm{~mm}$ to $30 \mathrm{~mm}$ that by doubling the height of $d 20$. Finally, choosing $d 30$ instead of $d 20 \times 2$ leads to $2 \%$ and $23 \%$ of increase for $B_{\mathrm{p}}$ and $B_{\mathrm{p}}{ }^{\mathrm{s}}$, respectively. The same tendency is reported experimentally at $20 \mathrm{~K}$, the increase of the maximal trapped field from $d 30$ to d20x2 is about $3 \%$.

From (3), the field-independent bulk critical current density $J_{\mathrm{c}}{ }^{\text {bulk }}$ can be calculated. For $d 20 \times 2$ at $20 \mathrm{~K}$, with $2.88 \mathrm{~T}$ of maximum trapped magnetic field at the surface of the sample, 
$J_{\mathrm{c}}^{\text {bulk }}$ is estimated around $48 \mathrm{kA} / \mathrm{cm}^{2}$. This value corresponds to the $J_{\mathrm{c}}(B)$ curve shown in Fig. 2 at $20 \mathrm{~K}$ and for $B=1.6 \mathrm{~T}$. Therefore, the mean value of trapped magnetic field in the sample volume is approximately equal to this value of $1.6 \mathrm{~T}$.

The values obtained here of the remanent trapped magnetic field measured at the center of the surface of the sample are a little bit lower than highest reported up to now in [12], i.e. $4.6 \mathrm{~T}, 3.3 \mathrm{~T}$ and $2 \mathrm{~T}$, at $15 \mathrm{~K}, 20 \mathrm{~K}$ and $25 \mathrm{~K}$, respectively. However, the obtained values of the maximal trapped magnetic field for $d 20 x 2$ of $3.63 \mathrm{~T}$ at $10 \mathrm{~K}, 2.88 \mathrm{~T}$ at $20 \mathrm{~K}$ $(2.98 \mathrm{~T}$ for $d 30$ ) and $2.03 \mathrm{~T}$ at $25 \mathrm{~K}$ are really interesting for the intended applications.

\section{CONCLUSION}

The trapped magnetic field, not only at the surface of a sample produced by SPS, but also inside a stack of two $\mathrm{MgB}_{2}$ samples, has been deeply investigated. The useful values of trapped magnetic field obtained at $20 \mathrm{~K}$ at the surface of the samples are really interesting for the intended applications. According to these values, to their very low density compared to other materials and to their ease of manufacturing, $\mathrm{MgB}_{2}$ bulks are promising materials for the applications of superconductors.

\section{ACKNOWLEDGMENT}

The authors would like to thank T. Karwoth and X. Zeng for their help during trapped field measurements at Saarland University.

\section{REFERENCES}

[1] J. Nagamatsu, N. Nakagawa, T. Muranaka, Y. Zenitani, and J. Akimitsu, "Superconductivity at $39 \mathrm{~K}$ in magnesium diboride," Nature, vol. 410, no. 6824, pp. 63-64, Mar. 2001.

[2] M. Muralidhar, Y. Fukumoto, A. Ishihara, K. Suzuki, M. Tomita, M. R. Koblischka, A. Yamamoto, and K. Kishio, "Recent developments in melt processed Gd-123 and $\mathrm{MgB}_{2}$ materials at RTRI," Phys. C Supercond., vol. 496, pp. 5-10, Jan. 2014.

[3] T. Prikhna, W. Gawalek, M. Eisterer, H. W. Weber, M. Monastyrov, V. Sokolovsky, J. Noudem, V. Moshchil, M. Karpets, V. Kovylaev, A. Borimskiy, V. Tkach, A. Kozyrev, R. Kuznietsov, J. Dellith, C. Shmidt, D. Litzkendorf, F. Karau, U. Dittrich, and M. Tomsic, "The effect of high-pressure synthesis on flux pinning in $\mathrm{MgB}_{2}$-based superconductors," Phys. C Supercond., vol. 479, pp. 111-114, Sep. 2012.

[4] J. G. Noudem, M. Aburras, P. Bernstein, X. Chaud, M. Muralidhar, and M. Murakami, "Development in processing of $\mathrm{MgB}_{2}$ cryo-magnet superconductors," J. Appl. Phys., vol. 116, no. 16, p. 163916, Oct. 2014.

[5] D.-X. Chen and R. B. Goldfarb, "Kim model for magnetization of type II superconductors," J. Appl. Phys., vol. 66, no. 6, pp. 2489-2500, Sep. 1989.

[6] A. Sanchez and C. Navau, "Critical-current density from magnetization loops of finite high-Tc superconductors," Supercond. Sci. Technol., vol. 14, no. 7, pp. 444-447, Jul. 2001.

[7] D. Batalu, G. Aldica, S. Popa, L. Miu, M. Enculescu, R. F. Negrea, I. Pasuk, and P. Badica, "High magnetic field enhancement of the critical current density by $\mathrm{Ge}, \mathrm{GeO}_{2}$ and $\mathrm{Ge}_{2} \mathrm{C}_{6} \mathrm{H}_{10} \mathrm{O}_{7}$ additions to $\mathrm{MgB}_{2}$," $\mathrm{Scr}$. Mater., vol. 82, pp. 61-64, Jul. 2014.

[8] P. Masson, J. Leveque, D. Netter, and A. Rezzoug, "Experimental study of a new kind of superconducting inductor," Appl. Supercond. IEEE Trans. On, vol. 13, no. 2, pp. 2239-2242, 2003.

[9] D. Netter, J. Leveque, E. Ailam, B. Douine, A. Rezzoug, and P. J. Masson, "Theoretical study of a new kind HTS motor," Appl. Supercond. IEEE Trans. On, vol. 15, no. 2, pp. 2186-2189, 2005.
[10] P. J. Masson and C. A. Luongo, "High power density superconducting motor for all-electric aircraft propulsion," Appl. Supercond. IEEE Trans. On, vol. 15, no. 2, pp. 2226-2229, 2005.

[11] G. Aldica, M. Burdusel, and P. Badica, "Trapped magnetic field in a (NdFeB)- $\left(\mathrm{MgB}_{2}\right)$ pair-type bulk magnet," Phys. C Supercond., vol. 505, pp. 18-23, Oct. 2014.

[12] G. Fuchs, W. Häßler, K. Nenkov, J. Scheiter, O. Perner, A. Handstein, T. Kanai, L. Schultz, and B. Holzapfel, "High trapped fields in bulk $\mathrm{MgB}_{2}$ prepared by hot-pressing of ball-milled precursor powder," Supercond. Sci. Technol., vol. 26, no. 12, p. 122002, Dec. 2013.

[13] V. Chabanenko, R. Puźniak, A. Nabiałek, S. Vasiliev, V. Rusakov, L. Huanqian, R. Szymczak, H. Szymczak, J. Jun, J. Karpiński, and V. Finkel, "Flux Jumps and H-T Diagram of Instability for $\mathrm{MgB}_{2}$," J. Low Temp. Phys., vol. 130, no. 3-4, pp. 175-191, Feb. 2003.

[14] A. Yamamoto, A. Ishihara, M. Tomita, and K. Kishio, "Permanent magnet with $\mathrm{MgB}_{2}$ bulk superconductor," Appl. Phys. Lett., vol. 105, no. 3, p. 032601, Jul. 2014.

[15] J. H. Durrell, C. E. J. Dancer, A. Dennis, Y. Shi, Z. Xu, A. M. Campbell, N. H. Babu, R. I. Todd, C. R. M. Grovenor, and D. A. Cardwell, "A trapped field of $>3 \mathrm{~T}$ in bulk $\mathrm{MgB}_{2}$ fabricated by uniaxial hot pressing," Supercond. Sci. Technol., vol. 25, no. 11, p. 112002, Nov. 2012.

[16] B. Douine, C.-H. Bonnard, F. Sirois, K. Berger, A. Kameni, and J. Leveque, "Determination of and -Value of HTS Pellets by Measurement and Simulation of Magnetic Field Penetration," IEEE Trans. Appl. Supercond., vol. 25, no. 4, pp. 1-8, Aug. 2015.

[17] E. H. Brandt, "Superconductor disks and cylinders in an axial magnetic field. I. Flux penetration and magnetization curves," Phys. Rev. B, vol. 58, no. 10, pp. 6506-6522, Sep. 1998.

[18] A. Forkl, "Magnetic flux distribution in single crystalline, ceramic and thin film high-Tc-superconductors," Phys. Scr., vol. 1993, no. T49A, p. 148, Jan. 1993. 